PROCEEDINGS OF THE

AMERICAN MATHEMATICAL SOCIETY

Volume 124, Number 12, December 1996, Pages 3755-3765

S 0002-9939(96)03616-7

\title{
THE MULTIPLIER OPERATORS ON THE WEIGHTED PRODUCT SPACES
}

\author{
LUNG-KEE CHEN AND DASHAN FAN
}

(Communicated by J. Marshall Ash)

\begin{abstract}
In this paper, we proved the boundedness of multiplier operators on the weighted $L^{p}$ product spaces.
\end{abstract}

Let $m(\xi)$ be a function on $R^{n}$ and let $f$ be a smooth function on $R^{n}$. Suppose

$$
\widehat{T_{m} f}(\xi)=m(\xi) \widehat{f}(\xi) .
$$

Then $T_{m} f$ is called a multiplier operator. It is well known that a multiplier operator is bounded on the weighted $L^{p}, 1<p<\infty$, spaces for some suitable weights if the function $m(\xi)$ satisfies Hörmander's condition

$$
\int_{s \leq|\xi| \leq 2 s}\left|\partial_{\xi}^{\alpha} m(\xi)\right|^{2} d \xi \leq s^{n-2|\alpha|}
$$

for $|\alpha| \leq[n / 2]+1$ (see [3, page 418]). The keys to proving the boundedness of multiplier operator in the weighted $L^{p}$ spaces are basically

(i) the Hardy-Littlewood maximal operator is bounded by the sharp function, more precisely,

$$
\int(M f(x))^{p} W(x) d x \leq C \int\left(f^{\#}(x)\right)^{p} W(x) d x
$$

where $W \in A_{p}, 1<p \leq \infty$, and

$$
f^{\#}(x)=\sup _{x \in Q} \frac{1}{|Q|} \int_{Q}\left|f(y)-f_{Q}\right| d y,
$$

$f_{Q}$ being the average of $f$ over cube $Q$ in $R^{n}$;

(ii) an estimate,

$$
\left(T_{m} f\right)^{\#}(x) \leq C\left(M|f|^{q}(x)\right)^{1 / q}
$$

for some $q>1$.

The purpose of this paper is to study the boundedness of multiplier operators on the weighted $L^{p}$ product spaces.

Denote

$$
\operatorname{osc}_{R} f=\inf _{\substack{f_{1} \\ f_{2}}}\left(\frac{1}{|R|} \int_{R}\left|f\left(x_{1}, x_{2}\right)-f_{1}\left(x_{1}\right)-f_{2}\left(x_{2}\right)\right|^{2} d x_{1} d x_{2}\right)^{1 / 2}
$$

Received by the editors May 25, 1995.

1991 Mathematics Subject Classification. Primary 42B15, 42B30.

(C)1996 American Mathematical Society 
where $R$ is any rectangle in $R^{n_{1}} \times R^{n_{2}}$ and the inf is taken over all functions $f_{1}$ and $f_{2}$ depending on the variables $x_{1}$ and $x_{2}$ respectively.

As in the "one dimensional case", if one defines the sharp function by

$$
f^{\#}(x)=\sup _{x \in R} \operatorname{osc}_{R} f,
$$

then one might expect to show that the strong maximal operator

$$
M_{s} f(x)=\sup _{\substack{t_{1}>0 \\ t_{2}>0}} \frac{1}{t_{1}^{n_{1}} t_{2}^{n_{2}}} \int_{\left|y_{1}\right| \leq t_{1}} \int_{\left|y_{2}\right| \leq t_{2}}\left|f\left(x_{1}-y_{1}, x_{2}-y_{2}\right)\right| d y_{1} d y_{2}
$$

is bounded by the sharp function. Unfortunately, such an observation is not true due to Carleson's counterexample [1]. To remove the difficulty on the sharp function in order to obtain an inequality similar to (1), R. Fefferman considered a sharp operator (see [2]) defined as follows.

Definition. Let $T$ be an $L^{2}$ bounded linear operator. Suppose there exists an operator $T^{\#}$ defined on positive locally square integrable functions which is monotone, i.e.

$$
T^{\#} f(x) \leq T^{\#} g(x)
$$

if $f(x) \leq g(x)$ for all $x \in R^{n_{1}} \times R^{n_{2}}$ such that $\operatorname{osc}_{R}(T f) \leq \gamma^{-\sigma} T^{\#} f(x)$ for all $x \in R, R$ a rectangle on $R^{n_{1}} \times R^{n_{2}}$, and for some $\sigma>0$, where $f$ is supported outside of the $\gamma$-fold dilation of $R, \gamma \geq 2$.

Based on this definition of a sharp operator, R. Fefferman [2] obtained the following inequality:

$$
\iint S^{2}(T f)(x) \phi(x) d x \leq C \iint\left[\left(I+T^{\#}\right)(|f|)\right]^{2}(x) M_{s}\left(M_{s}\left(M_{s}\left(M_{s}(\phi)\right)\right)\right)(x) d x
$$

where $I$ denotes the identity operator and $S$ is the area function defined on the product spaces. Using this inequality, he obtained the following theorem, which we will apply in this paper.

Theorem A ([2]). If $T$ is a bounded linear operator on $L^{2}\left(R^{n_{1}} \times R^{n_{2}}\right)$ whose sharp operator is

$$
T^{\#} f=M_{s}\left(f^{2}\right)^{1 / 2},
$$

then for $p>2$

$$
\int_{R^{n_{1}} \times R^{n_{2}}}|T f|^{p} W \leq C \int_{R^{n_{1}} \times R^{n_{2}}}|f|^{p} W
$$

whenever $W \in A_{p / 2}\left(R^{n_{1}} \times R^{n_{2}}\right)$.

Proof. See [2, page 123].

In this paper, we will prove the following Theorem. 
Theorem. Let $m\left(\xi_{1}, \xi_{2}\right)$ be a function and $m \in C^{p_{1}}\left(R^{n_{1}} \backslash\{0\}\right) \times C^{p_{2}}\left(R^{n_{2}} \backslash\{0\}\right)$, where $p_{1}=\left[n_{1} / 2\right]+1$, and $p_{2}=\left[n_{2} / 2\right]+1$. Suppose

$$
\begin{aligned}
& \int_{\left|\xi_{1}\right| \approx s_{1}} \int_{\left|\xi_{2}\right| \approx s_{2}}\left|\partial_{\xi_{1}}^{\alpha_{1}} \partial_{\xi_{2}}^{\alpha_{2}} m\left(\xi_{1}, \xi_{2}\right)\right|^{2} d \xi_{1} d \xi_{2} \leq C s_{1}^{-2\left|\alpha_{1}\right|+n_{1}} s_{2}^{-2\left|\alpha_{2}\right|+n_{2}} ; \\
& \sup _{\xi_{2}} \int_{\left|\xi_{1}\right| \approx s_{1}}\left|\partial_{\xi_{1}}^{\alpha_{1}} m\left(\xi_{1}, \xi_{2}\right)\right|^{2} d \xi_{1} d \xi_{2} \leq C s_{1}^{-2\left|\alpha_{1}\right|+n_{1}} ; \\
& \sup _{\xi_{1}} \int_{\left|\xi_{2}\right| \approx s_{2}}\left|\partial_{\xi_{2}}^{\alpha_{2}} m\left(\xi_{1}, \xi_{2}\right)\right|^{2} d \xi_{1} d \xi_{2} \leq C s_{2}^{-2\left|\alpha_{2}\right|+n_{2}}
\end{aligned}
$$

for every $\left|\alpha_{1}\right| \leq p_{1}$ and $\left|\alpha_{2}\right| \leq p_{2}$, where $\left|\xi_{i}\right| \approx s_{i}$ signifies that $s_{i} \leq\left|\xi_{i}\right| \leq 2 s_{i}$.

Then i) for $2<p<\infty$,

$$
\int\left|T_{m} f\right|^{p} W \leq C \int|f|^{p} W
$$

whenever $W \in A_{p / 2}\left(R^{n_{1}} \times R^{n_{2}}\right)$; and ii) for $1<p<2$,

$$
\int\left|T_{m} f\right|^{p} W \leq C \int|f|^{p} W
$$

whenever $W^{\frac{2}{2-p}} \in A_{\frac{p}{2-p}}\left(R^{n_{1}} \times R^{n_{2}}\right)$.

Remark. The weighted norm inequality for $p=2$ can be obtained by using the interpolation theorem.

Proof. From Theorem A, we need to show that the sharp operator $T^{\#} f=$ $M_{s}\left(f^{2}\right)^{1 / 2}$. Then (i) of our Theorem follows from Theorem A.

Let us take a smooth function $\phi$ on $R^{1}$ whose Fourier transform $\widehat{\phi}(t)$ has compact support $\{1 / 2<|t|<2\}$ such that $\sum \widehat{\phi}\left(2^{-j}|t|\right)=1$ for all $t \neq 0$. Let

$$
m_{i, j}\left(\xi_{1}, \xi_{2}\right)=m\left(\xi_{1}, \xi_{2}\right) \widehat{\phi}\left(2^{-i}\left|\xi_{1}\right|\right) \widehat{\phi}\left(2^{-j}\left|\xi_{2}\right|\right)
$$

and

$$
\widehat{T}_{i, j} f\left(\xi_{1}, \xi_{2}\right)=m_{i, j}\left(\xi_{1}, \xi_{2}\right) \widehat{f}\left(\xi_{1}, \xi_{2}\right) \equiv\left(\widehat{\left(k_{i, j} * f\right.}\right)\left(\xi_{1}, \xi_{2}\right) .
$$

It is clear that $T f=\sum_{i, j} T_{i, j} f$.

To prove $T^{\#} f(x)=\left(M_{s} f^{2}(x)\right)^{1 / 2}$, one needs to estimate, for every rectangle $R$,

$$
\operatorname{Osc}_{R}(T f)(x) \leq C \gamma^{-\delta}\left(M_{s} f^{2}(x)\right)^{1 / 2}
$$

for every $x \in R$ where $f$ is supported outside of the $\gamma$-fold dilation of the rectangle $R, \gamma \geq 2$, i.e. supp $f \subset \tilde{R}_{\gamma}^{c}$. By the homogeneity of multiplier operators, it suffices to assume $R$ is the unit square. Since the estimates are translation invariant, we may assume the center of $R$ is at the origin. Let us write a function $f$, supp $f \subset \tilde{R}_{\gamma}^{c}$, as the sum of the functions $g+h+G$ where

$$
\begin{aligned}
& \text { support of } g \subset{ }^{c} \tilde{R}_{\gamma}^{1} \equiv\left\{\left|y_{1}\right|>\gamma,\left|y_{2}\right| \leq \gamma\right\} \\
& \text { support of } h \subset{ }^{c} \tilde{R}_{\gamma}^{3} \equiv\left\{\left|y_{1}\right|>\gamma,\left|y_{2}\right|>\gamma\right\} \\
& \text { support of } G \subset{ }^{c} \tilde{R}_{\gamma}^{2} \equiv\left\{\left|y_{1}\right| \leq \gamma,\left|y_{2}\right|>\gamma\right\}
\end{aligned}
$$


Without loss of generality it suffices to show (3) for a function $f=g+h$ where

$$
\begin{aligned}
& \operatorname{supp} g \subset{ }^{c} \tilde{R}_{\gamma}^{1} \subset\left\{\left|y_{1}\right|>\gamma,\left|y_{2}\right| \leq 2\right\} ; \\
& \operatorname{supp} h \subset{ }^{c} \tilde{R}_{\gamma}^{3} \subset\left\{\left|y_{1}\right|>\gamma,\left|y_{2}\right|>2\right\} .
\end{aligned}
$$

We are going to estimate

$$
\operatorname{osc}_{R}(T g)(x) \leq C \gamma^{-\sigma}\left(M_{s} g^{2}(x)\right)^{1 / 2} \text { and } \operatorname{osc}_{R}(T h)(x) \leq C \gamma^{-\sigma}\left(M_{s} h^{2}(x)\right)^{1 / 2},
$$

since $\operatorname{osc}_{R} T f \leq \operatorname{osc}_{R} T g+\operatorname{osc}_{R} T h$. Let us write

$$
\begin{aligned}
\operatorname{osc}_{R}(T f) \leq & \sum_{i \geq 0} \operatorname{osc}_{R}\left(\sum_{j} T_{i, j} g\right)+\sum_{i<0} \operatorname{osc}_{R}\left(\sum_{j} T_{i, j} g\right) \\
& +\sum_{i \geq 0} \sum_{j \geq 0} \operatorname{osc}_{R} T_{i, j} h+\sum_{i \geq 0} \sum_{j \leq 0} \operatorname{osc}_{R} T_{i, j} h \\
& +\sum_{i<0} \sum_{j \geq 0} \operatorname{osc}_{R} T_{i, j} h+\sum_{i<0} \sum_{j<0} \operatorname{osc}_{R} T_{i, j} h \\
\equiv & I+I I+I I I+I V+V+V I .
\end{aligned}
$$

We will estimate that the first two terms are dominated by $\gamma^{-\sigma}\left(M_{s} g^{2}(x)\right)^{1 / 2}$ and the last four terms are dominated by $\gamma^{-\sigma}\left(M_{s} h^{2}(x)\right)^{1 / 2}$ for every $x \in R$. Denote $\sum_{j} T_{i, j} g \equiv T_{i} g$ and write

$$
\begin{aligned}
& I=\sum_{i \geq 0} \operatorname{osc}_{R}\left(T_{i} g\right) \\
& \leq \sum_{i \geq 0}\left(\frac{1}{|R|} \int_{R}\left|T_{i} g\left(x_{1}, x_{2}\right)\right|^{2} d x_{1} d x_{2}\right)^{1 / 2} \\
&=\sum_{i \geq 0}\left(\int_{\left|x_{1}\right| \leq 1} \int_{\left|x_{2}\right| \leq 1} \mid \int_{\left|y_{2}\right|<2} \int_{\left|y_{1}\right| \geq \gamma} K_{i}\left(x_{1}-y_{1}, x_{2}-y_{2}\right) g\left(y_{1}, y_{2}\right)\right. \\
& \leq \sum_{i \geq 0} \sum_{2^{k_{1}} \geq \gamma / 2}\left(\int_{\left|x_{1}\right| \leq 1} \int_{\left|x_{2}\right| \leq 1}\left|\int_{\left|y_{2}\right|<2} \int_{\left|y_{1}\right| \approx 2^{k_{1}}} d y_{2}\right|^{2} d x_{2} d x_{1}\right)^{1 / 2} \\
&\left.\left.\cdot K_{i}\left(x_{1}-y_{1}, x_{2}-y_{2}\right) g\left(y_{1}, y_{2}\right) d y_{1} d y_{2}\right|^{2} d x_{2} d x_{1}\right)^{1 / 2} .
\end{aligned}
$$

Since

$$
\int_{\left|y_{1}\right| \approx 2^{k_{1}}} \int_{\left|y_{2}\right|<2} K_{i}\left(x_{1}-y_{1}, x_{2}-y_{2}\right) g\left(y_{1}, y_{2}\right) d y_{2} d y_{1}
$$

is a convolution operator in the variable $y_{2}$, applying Plancherel's Theorem for the variable $x_{2}$ on (4), one has

$$
I \leq \sum_{i \geq 0} \sum_{2^{k_{1}} \geq \gamma / 2}\left(\int_{\left|x_{1}\right| \leq 1} \int_{\xi_{2}}\left|\int_{\left|y_{1}\right| \approx 2^{k_{1}}} \widehat{K}_{i}^{2}\left(x_{1}-y_{1}, \xi_{2}\right) \widehat{g}^{2}\left(y_{1}, \xi_{2}\right) d y_{1}\right|^{2} d \xi_{2} d x_{1}\right)^{1 / 2}
$$


where $\wedge^{\wedge}$ denotes the Fourier transform on the second variable. For $\left|x_{1}\right| \leq 1$, $\left|y_{1}\right| \geq \gamma, \gamma \geq 2,\left|y_{1}\right| \approx 2^{k_{1}}$ then $\left|x_{1}-y_{1}\right| \approx 2^{k_{1}}$. Hence

$$
\begin{array}{r}
I \leq \sum_{i \geq 0} \sum_{2^{k_{1}} \geq \gamma / 2} 2^{-k_{1} p_{1}}\left(\int_{\left|x_{1}\right| \leq 1} \int_{\xi_{2}}\left|\int_{\left|y_{1}\right| \approx 2^{k_{1}}}\right| x_{1}-\left.y_{1}\right|^{p_{1}} \widehat{K}_{i}^{2}\left(x_{1}-y_{1}, \xi_{2}\right)\right. \\
\left.\left.\cdot \widehat{g}^{2}\left(y_{1}, \xi_{2}\right) d y_{1}\right|^{2} d \xi_{2} d x_{1}\right)^{1 / 2} .
\end{array}
$$

By Hölder's inequality and changing variable $y_{1}$,

$$
\begin{aligned}
& I \leq \sum_{i \geq 0} \sum_{2^{k_{1}} \geq \gamma / 2} 2^{-k_{1} p_{1}}\left(\int_{\left|x_{1}\right| \leq 1} \int_{\xi_{2}} \int|| x_{1}-\left.\left.y_{1}\right|^{p_{1}} \widehat{K}_{i}^{2}\left(x_{1}-y_{1}, \xi_{2}\right)\right|^{2} d y_{1}\right. \\
& \left.\cdot \int_{\left|y_{1}\right| \approx 2^{k_{1}}}\left|\widehat{g}^{2}\left(y_{1}, \xi_{2}\right)\right|^{2} d y_{1} d \xi_{2} d x_{1}\right)^{1 / 2} \\
& \leq \sum_{i \geq 0} \sum_{2^{k_{1}} \geq \gamma / 2} 2^{-k_{1} p_{1}}\left(\left.\left.\sup _{\xi_{2}} \int|| y_{1}\right|^{p_{1}} \widehat{K}_{i}^{2}\left(y_{1}, \xi_{2}\right)\right|^{2} d y_{1}\right)^{1 / 2} \\
& \cdot\left(\iint_{\left|y_{1}\right| \approx 2^{k_{1}}}\left|\widehat{g}^{2}\left(y_{1}, \xi_{2}\right)\right|^{2} d y_{1} d \xi_{2}\right)^{1 / 2} \\
& \leq \sum_{i \geq 0} \sum_{2^{k_{1}} \geq \gamma / 2} 2^{k_{1}\left(-p_{1}+n_{1} / 2\right)}\left(\sup _{\xi_{2}} \sum_{\left|\alpha_{1}\right|=p_{1}} \int\left|\partial_{\xi_{1}}^{\alpha_{1}} m_{i}\left(\xi_{1}, \xi_{2}\right)\right|^{2} d \xi_{1}\right)^{1 / 2} \\
& \cdot\left(\frac{1}{2^{k_{1} n_{1}}} \int_{\left|y_{2}\right| \leq 2} \int_{\left|y_{1}\right| \approx 2^{k_{1}}}\left|g\left(y_{1}, y_{2}\right)\right|^{2} d y_{1} d y_{2}\right)^{1 / 2}
\end{aligned}
$$

where the last inequality is obtained by applying Plancherel's Theorem to both integrals and the support of $g$ is contained by $\left\{\left|y_{1}\right|>\gamma,\left|y_{2}\right| \leq 2\right\}$. Hence, by the hypothesis (2) and $-p_{1}+n_{1} / 2<0$,

$$
I \leq C \sum_{i \geq 0} \gamma^{-\sigma}\left(M_{s} g^{2}(0)\right)^{1 / 2} 2^{i\left(-p_{1}+n_{1} / 2\right)} \leq C \gamma^{-\sigma}\left(M_{s} g^{2}(0)\right)^{1 / 2}
$$

For estimating II, we write

$$
\begin{aligned}
I I & =\sum_{i<0} \operatorname{osc}_{R}\left(\sum_{j} T_{i, j} g\right)=\sum_{i<0} \operatorname{osc}_{R}\left(T_{i} g\right) \\
& \leq \sum_{i<0}\left(\frac{1}{|R|} \int_{R}\left|T_{i} g\left(x_{1}, x_{2}\right)-T_{i} g\left(0, x_{2}\right)\right|^{2} d x_{1} d x_{2}\right)^{1 / 2} \\
& =C \sum_{i<0}\left(\int_{R} \mid \int_{c_{R_{\gamma}^{\prime}}^{1}}\left(K_{i}\left(x_{1}-y_{1}, x_{2}-y_{2}\right)-K_{i}\left(0-y_{1}, x_{2}-y_{2}\right)\right)\right. \\
& \leq C \sum_{i<0} \sum_{2^{k_{1}} \geq \gamma / 2}\left(\int_{R} \mid \int_{\left|y_{1}\right| \approx 2^{k_{1}}} \int_{\left|y_{2}\right| \leq 2}\left(\left.K_{i}\left(x_{1}-y_{1}, y_{2}\right) d y_{1} d y_{2}\right|^{2} d x_{2} d x_{1}\right)^{1 / 2}\right. \\
& \left.\left.\quad-K_{i}\left(0-y_{1}, x_{2}-y_{2}\right)\right)\left.g\left(y_{1}, y_{2}\right) d y_{1} d y_{2}\right|^{2} d x_{2} d x_{1}\right)^{1 / 2} .
\end{aligned}
$$


Here, we follow the same procedures as we did in proving I, applying Plancherel's Theorem for the variable $x_{2}$,

$$
\begin{aligned}
& I I \leq C \sum_{i<0} \sum_{2^{k_{1} \geq \gamma / 2}}\left(\int_{\left|x_{1}\right| \leq 1} \int_{\xi_{2}} \mid \int_{\left|y_{1}\right| \approx 2^{k_{1}}}\left(\widehat{K}_{i}^{2}\left(x_{1}-y_{1}, \xi_{2}\right)-\widehat{K}_{i}^{2}\left(0-y_{1}, \xi_{2}\right)\right)\right. \\
&=C \sum_{i<0} \sum_{2^{k_{1}} \geq \gamma / 2}\left(\int_{\left|x_{1}\right| \leq 1} \int_{\xi_{2}} \mid \int_{\left|y_{1}\right| \approx 2^{k_{1}}} \int_{0}^{1} x_{1} \partial_{y_{1}} \widehat{K}_{i}^{2}\left(x_{1} s_{1}-\left.y_{1}\right|^{2} d \xi_{2} d x_{1}\right)^{1 / 2}\right. \\
& \leq C \sum_{i<0} \sum_{2^{k_{1}} \geq \gamma / 2}\left(\left.\int_{0}^{1} \int_{\left|x_{1}\right| \leq 1} \int_{\xi_{2}}\left(\int_{\left|y_{1}\right| \approx 2^{k_{1}}} \mid \partial_{y_{1}} \widehat{K}_{i}^{2}\left(\left.x_{1}\left(y_{1}, \xi_{2}\right) d s_{1} d y_{1}\right|^{2} d \xi_{2} d x_{1}\right)^{1 / 2} \xi_{2}\right)\right|^{2} d y_{1}\right) \\
&\left.\leq C \sum_{i<0} \sum_{2^{k_{1} \geq \gamma / 2}} 2^{k_{1}\left(-p_{1}+\epsilon_{1}+n_{1} / 2\right)}\left[\left.\int_{\left|y_{1}\right| \approx 2^{k_{1}}}^{1} \int_{\left|x_{1}\right| \leq 1} \sup _{\xi_{2}}\left(\int_{\left|y_{1}\right| \approx 2^{k_{1}}}|| y_{1}, \xi_{2}\right)\right|^{2} d y_{1}\right) d \xi_{2} d x_{1} d s_{1}\right)\left.^{1 / 2} y_{1}\right|^{p_{1}-\epsilon_{1}} \\
&\left.\cdot\left(\frac{1}{2^{k_{1} n_{1}}} \iint_{\left|y_{1}\right| \approx 2^{k_{1}}}\left|\widehat{g}^{2}\left(y_{1}, \xi_{2}\right)\right|^{2} d y_{1} d \xi_{2}\right) d x_{1} d s_{1}\right]^{1 / 2} \cdot
\end{aligned}
$$

Taking a very small $\epsilon_{1}>0$ such that $-p_{1}+\epsilon_{1}+n_{1} / 2<0$, changing variable (i.e. $\left.x_{1} s_{1}-y_{1} \rightarrow y_{1}\right)$ in the integral in the first parentheses and applying Plancherel's Theorem for the integral in the second parentheses, one has

$$
\begin{aligned}
I I & \leq C \gamma^{-\sigma} \sum_{i<0} \sup _{\xi_{2}}\left(\left.\left.\int|| y_{1}\right|^{-\epsilon_{1}}\left|y_{1}\right|^{p_{1}} \partial_{y_{1}} \widehat{K}_{i}^{2}\left(y_{1}, \xi_{2}\right)\right|^{2} d y_{1}\right)^{1 / 2}\left(M_{s} g^{2}(0)\right)^{1 / 2} \\
& \leq C \gamma^{-\sigma}\left(M_{s} g^{2}(0)\right)^{1 / 2} \sum_{i<0} \sup _{\xi_{2}} \sum_{\left|\alpha_{1}\right|=p_{1}}\left(\left.\left.\int|| \xi_{1}\right|^{-n_{1}+\epsilon_{1}} *^{1} \partial_{\xi_{1}}^{\alpha_{1}}\left(\xi_{1} m_{i}\left(\xi_{1}, \xi_{2}\right)\right)\right|^{2} d \xi_{1}\right)^{1 / 2}
\end{aligned}
$$

for some $\sigma>0$, where $*^{1}$ is the convolution operator on the first variable. By fractional integration,

$$
I I \leq C \gamma^{-\sigma}\left(M_{s} g^{2}(0)\right)^{1 / 2} \sum_{i<0} \sum_{\left|\alpha_{1}\right|=p_{1}} \sup _{\xi_{2}}\left(\int\left|\partial_{\xi_{1}}^{\alpha_{1}}\left(\xi_{1} m_{i}\left(\xi_{1}, \xi_{2}\right)\right)\right|^{q} d \xi_{1}\right)^{1 / q}
$$


where $1 / q=1 / 2+\epsilon_{1} / n_{1}$ (clearly $q<2$ ). By Hölder's inequality,

$$
\begin{aligned}
I I & \leq C \gamma^{-\sigma}\left(M_{s} g^{2}(0)\right)^{1 / 2} \sum_{i<0} \sum_{\left|\alpha_{1}\right|=p_{1}} 2^{i n_{1}(1 / q-1 / 2)} \sup _{\xi_{2}}\left(\int\left|\partial_{\xi_{1}}^{\alpha_{1}}\left(\xi_{1} m_{i}\left(\xi_{1}, \xi_{2}\right)\right)\right|^{2} d \xi_{1}\right)^{1 / 2} \\
& \leq C \gamma^{-\sigma}\left(M_{s} g^{2}(0)\right)^{1 / 2} \sum_{i<0} 2^{i\left(-p_{1}+1+n_{1} / q\right)} \\
& \leq C \gamma^{-\sigma}\left(M_{s} g^{2}(0)\right)^{1 / 2}
\end{aligned}
$$

(since $\epsilon_{1}>0$ then $-p_{1}+1+n_{1} / q>0$ ).

For estimating III, we write

$$
\begin{aligned}
& \left|T_{i, j} h\left(x_{1}, x_{2}\right)\right| \\
& \leq \sum_{k_{2} \geq 1} \sum_{2^{k_{1}} \geq \gamma / 2} \int_{\left|y_{1}\right| \approx 2^{k_{1}}} \int_{\left|y_{2}\right| \approx 2^{k_{2}}}\left|K_{i, j}\left(x_{1}-y_{1}, x_{2}-y_{2}\right) h\left(y_{1}, y_{2}\right)\right| d y_{1} d y_{2} \\
& \leq \sum_{k_{2} \geq 1} \sum_{2^{k_{1}} \geq \gamma / 2}\left(2^{k_{1} n_{1}} 2^{k_{2} n_{2}} \int_{\left|y_{1}\right| \approx 2^{k_{1}}} \int_{\left|y_{2}\right| \approx 2^{k_{2}}}\left|K_{i, j}\left(x_{1}-y_{1}, x_{2}-y_{2}\right)\right|^{2} d y_{1} d y_{2}\right)^{1 / 2} \\
& \cdot\left(\frac{1}{2^{k_{1} n_{1}} 2^{k_{2} n_{2}}} \int_{\left|y_{1}\right| \approx 2^{k_{1}}} \int_{\left|y_{2}\right| \approx 2^{k_{2}}}|h|^{2} d y_{1} d y_{2}\right)^{1 / 2} \\
& \leq C\left(M_{s} h^{2}(0)\right)^{1 / 2} \sum_{k_{2} \geq 1} \sum_{2^{k_{1}} \geq \gamma / 2} 2^{k_{1}\left(-p_{1}+n_{1} / 2\right)} 2^{k_{2}\left(-p_{2}+n_{2} / 2\right)} \\
& \cdot\left(\iint|| x_{1}-\left.\left.y_{1}\right|^{p_{1}}\left|x_{2}-y_{2}\right|^{p_{2}} K_{i, j}\left(x_{1}-y_{1}, x_{2}-y_{2}\right)\right|^{2} d y_{1} d y_{2}\right)^{1 / 2} .
\end{aligned}
$$

As before, applying a change of variable and Plancherel's Theorem,

$$
\begin{aligned}
& \left|T_{i, j} h\left(x_{1}, x_{2}\right)\right| \\
& \quad \leq C \gamma^{-\sigma}\left(M_{s} h^{2}(0)\right)^{1 / 2} \sum_{\left|\alpha_{1}\right|=p_{1}\left|\alpha_{2}\right|=p_{2}} \sum\left(\iint\left|\partial_{\xi_{1}}^{\alpha_{1}} \partial_{\xi_{2}}^{\alpha_{2}} m_{i, j}\left(\xi_{1}, \xi_{2}\right)\right|^{2} d \xi_{1} \xi_{2}\right)^{1 / 2} \\
& \quad \leq C \gamma^{-\sigma}\left(M_{s} h^{2}(0)\right)^{1 / 2} 2^{i\left(-p_{1}+n_{1} / 2\right)} 2^{j\left(-p_{2}+n_{2} / 2\right)} .
\end{aligned}
$$

Hence

$$
I I I=\sum_{i \geq 0} \sum_{j \geq 0} \operatorname{osc}_{R} T_{i, j} h \leq C \gamma^{-\sigma}\left(M_{s} h^{2}(0)\right)^{1 / 2} .
$$


Since the estimates for IV and V are similar, we estimate only term V. First let us write

$$
\begin{aligned}
& \left|T_{i, j} h\left(x_{1}, x_{2}\right)-T_{i, j} h\left(0, x_{2}\right)\right| \\
& \leq \sum_{k_{2} \geq 1} \sum_{2^{k_{1}} \geq \gamma / 2} \int_{\left|y_{1}\right| \approx 2^{k_{1}}} \int_{\left|y_{2}\right| \approx 2^{k_{2}}}\left|K_{i, j}\left(x_{1}-y_{1}, x_{2}-y_{2}\right)-K_{i, j}\left(0-y_{1}, x_{2}-y_{2}\right)\right| \\
& \leq \sum_{k_{2} \geq 1} \sum_{2^{k_{1} \geq \gamma / 2}} 2^{k_{1} n_{1} / 2} 2^{k_{2} n_{2} / 2}\left(\int_{\left|y_{1}\right| \approx 2^{k_{1}}} \int_{\left|y_{2}\right| \approx 2^{k_{2}}}\left|K_{i, j}\left(x_{1}, y_{2}\right)\right| d y_{1} d y_{2}, y_{1}, x_{2}-y_{2}\right) \\
& \left.\left.\quad-\left.K_{i, j}\left(0-y_{1}, x_{2}-y_{2}\right)\right|^{2} d y_{1} d y_{2}\right)^{1 / 2}\left(M_{s} h^{2}(0)\right)^{1 / 2} \cdot d y_{1} d y_{2} d s\right)^{1 / 2} \\
& \leq \sum_{k_{2} \geq 1} \sum_{2^{k_{1} \geq \gamma / 2}} 2^{k_{1} n_{1} / 2} 2^{k_{2} n_{2} / 2}\left(M_{s} h^{2}(0)\right)^{1 / 2}\left(\int_{0}^{1} \iint\left|\partial_{y_{1}} K_{i, j}\left(x_{1} s_{1}-y_{1}, x_{2}-y_{2}\right)\right|^{2}\right. \\
& \approx \sum_{k_{2} \geq 1} \sum_{2^{k_{1} \geq \gamma / 2}} 2^{k_{1}\left(-p_{1}+\epsilon_{1}+n_{1} / 2\right)} 2^{k_{2}\left(-p_{2}+n_{2} / 2\right)}\left(M_{s} h^{2}(0)\right)^{1 / 2}\left(\int_{0}^{1} \iint\right. \\
& \left.\cdot|| x_{1} s_{1}-\left.\left.y_{1}\right|^{p_{1}-\epsilon_{1}}\left|x_{2}-y_{2}\right|^{p_{2}} \partial_{y_{1}} K_{i, j}\left(x_{1} s_{1}-y_{1}, x_{2}-y_{2}\right)\right|^{2} d y_{1} d y_{2} d s\right)^{1 / 2} .
\end{aligned}
$$

Taking a positive small $\epsilon_{1}$, changing variables, applying Plancherel's Theorem and fractional integration, we get

$$
\begin{aligned}
& \left|T_{i, j} h\left(x_{1}, x_{2}\right)-T_{i, j} h\left(0, x_{2}\right)\right| \\
& \leq C \gamma^{-\sigma}\left(M_{s} h^{2}(0)\right)^{1 / 2} \\
& \quad \cdot \sum_{\left|\alpha_{1}\right|=p_{1}} \sum_{\left|\alpha_{2}\right|=p_{2}}\left(\left.\left.\iint|| \xi_{1}\right|^{-n_{1}+\epsilon_{1}} *^{1} \partial_{\xi_{1}}^{\alpha_{1}} \partial_{\xi_{2}}^{\alpha_{2}}\left(\xi_{1} m_{i, j}\left(\xi_{1}, \xi_{2}\right)\right)\right|^{2} d \xi_{1} d \xi_{2}\right)^{1 / 2} \\
& \leq C \gamma^{-\sigma}\left(M_{s} h^{2}(0)\right)^{1 / 2} \\
& \quad \cdot \sum_{\left|\alpha_{1}\right|=p_{1}} \sum_{\left|\alpha_{2}\right|=p_{2}}\left(\int\left(\left.\int\left|\partial_{\xi_{1}}^{\alpha_{1}} \partial_{\xi_{2}}^{\alpha_{2}}\right|\left(\xi_{1} m_{i, j}\left(\xi_{1}, \xi_{2}\right)\right)\right|^{q} d \xi_{1}\right)^{2 / q} d \xi_{2}\right)^{1 / 2}
\end{aligned}
$$

where $1 / q=1 / 2+\epsilon_{1} / n_{1}$. By Hölder's inequality and the hypothesis (2),

$$
\begin{aligned}
& \left|T_{i, j} h\left(x_{1}, x_{2}\right)-T_{i, j} h\left(0, x_{2}\right)\right| \\
& \leq C \gamma^{-\sigma}\left(M_{s} h^{2}(0)\right)^{1 / 2} 2^{i n_{1}(1 / q-1 / 2)}\left(\iint\left|\partial_{\xi_{1}}^{\alpha_{1}} \partial_{\xi_{2}}^{\alpha_{2}}\left(\xi_{1} m_{i, j}\left(\xi_{1}, \xi_{2}\right)\right)\right|^{2} d \xi_{1} d \xi_{2}\right)^{1 / 2} \\
& \leq C \gamma^{-\sigma}\left(M_{s} h^{2}(0)\right)^{1 / 2} 2^{i\left(-p_{1}+1+n_{1} / q\right)} 2^{j\left(-p_{2}+n_{2} / 2\right)} .
\end{aligned}
$$

Since $-p_{1}+1+n_{1} / q>0$ for positive small $\epsilon_{1}$ and $-p_{2}+n_{2} / 2<0$, we have

$$
V=\sum_{i<0} \sum_{j \geq 0} \operatorname{osc}_{R} T_{i, j} h \leq C \gamma^{-\sigma}\left(M_{s} h^{2}(0)\right)^{1 / 2}
$$


For the last term VI, we consider

$$
\begin{aligned}
& \left|T_{i, j} h\left(x_{1}, x_{2}\right)-T_{i, j} h\left(0, x_{2}\right)-T_{i, j} h\left(x_{1}, 0\right)+T_{i, j} h(0,0)\right| \\
& \leq \sum_{k_{2} \geq 1} \sum_{2^{k_{1}} \geq \gamma / 2} \int_{\left|y_{1}\right| \approx 2^{k_{1}}} \int_{\left|y_{2}\right| \approx 2^{k_{2}}}\left(K_{i, j}\left(x_{1}-y_{1}, x_{2}-y_{2}\right)-K_{i, j}\left(-y_{1}, x_{2}-y_{2}\right)\right. \\
& \left.\quad-K_{i, j}\left(x_{2}-y_{1},-y_{2}\right)+K_{i, j}\left(-y_{1},-y_{2}\right)\right) h\left(y_{1}, y_{2}\right) d y_{1} d y_{2} \\
& \leq \sum_{k_{2} \geq 1} \sum_{2^{k_{1}} \geq \gamma / 2}\left(2^{k_{1} n_{1}} 2^{k_{2} n_{2}} \int_{\left|y_{1}\right| \approx 2^{k_{1}}} \int_{\left|y_{2}\right| \approx 2^{k_{2}}} \mid K_{i, j}\left(x_{1}-y_{1}, x_{2}-y_{2}\right)\right. \\
& \left.-K_{i j}\left(-y_{1}, x_{2}-y_{2}\right)-K_{i j}\left(x_{1}-y_{1},-y_{2}\right)+\left.K_{i j}\left(-y_{1},-y_{2}\right)\right|^{2} d y_{1} d y_{2}\right)^{1 / 2} \\
& \cdot\left(\frac{1}{2^{k_{1} n_{1}} 2^{k_{2} n_{2}}} \int_{\left|y_{1}\right| \approx 2^{k_{1}}} \int_{\left|y_{2}\right| \approx 2^{k_{2}}}|h|^{2} d y_{1} d y_{2}\right)^{1 / 2} \cdot
\end{aligned}
$$

By the Taylor formula and Hölder's inequality,

$$
\begin{aligned}
& \left|T_{i, j} h\left(x_{1}, x_{2}\right)-T_{i, j} h\left(0, x_{2}\right)-T_{i, j} h\left(x_{1}, 0\right)+T_{i, j} h(0,0)\right| \\
& \leq \sum_{k_{2} \geq 1} \sum_{2^{k_{1}} \geq \gamma / 2}\left(M_{s} h^{2}(0)\right)^{1 / 2}\left(2^{k_{1} n_{1}} 2^{k_{2} n_{2}} \int_{0}^{1} \int_{0}^{1} \int_{\left|y_{1}\right| \approx 2^{k_{1}}} \int_{\left|y_{2}\right| \approx 2^{k_{2}}}\right. \\
& \left.\cdot\left|\partial_{y_{1}} \partial_{y_{2}} K_{i j}\left(x_{1} s_{1}-y_{1}, x_{2} s_{2}-y_{2}\right)\right|^{2} d y_{1} d y_{2} d s_{1} d s_{2}\right)^{1 / 2} \\
& \approx C\left(M_{s} h^{2}(0)\right)^{1 / 2} \sum_{k_{2} \geq 1} \sum_{2^{k_{1}} \geq \gamma / 2} 2^{k_{1}\left(-p_{1}+\epsilon_{1}+n_{1} / 2\right)} 2^{k_{2}\left(-p_{2}+\epsilon_{2}+n_{2} / 2\right)} \\
& \cdot\left(\int_{0}^{1} \int_{0}^{1} \int_{\left|y_{1}\right| \approx 2^{k_{1}}} \int_{\left|y_{2}\right| \approx 2^{k_{2}}}|| x_{1} s_{1}-\left.y_{1}\right|^{p_{1}-\epsilon_{1}}\left|x_{2} s_{2}-y_{2}\right|^{p_{2}-\epsilon_{2}}\right. \\
& \left.\left.\cdot \partial_{y_{1}} \partial_{y_{2}} K_{i j}\left(x_{1} s_{1}-y_{1}, x_{2} s_{2}-y_{2}\right)\right|^{2} d y_{1} d y_{2} d s_{1} d s_{2}\right)^{1 / 2} \\
& \leq\left. C \gamma^{-\sigma}\left(M_{s} h^{2}(0)\right)^{1 / 2} \iint|| y_{1}\right|^{-\epsilon_{1}}\left|y_{2}\right|^{-\epsilon_{2}}\left|y_{1}\right|^{p_{1}}\left|y_{2}\right|^{p_{2}} \\
& \left.\left.\cdot \partial_{y_{1}} \partial_{y_{2}} K_{i j}\left(y_{1}, y_{2}\right)\right|^{2} d y_{1} d y_{2}\right)^{1 / 2} \\
& \leq C \gamma^{-\sigma}\left(M_{s} h^{2}(0)\right)^{1 / 2} \sum_{\left|\alpha_{1}\right|=p_{1}} \sum_{\left|\alpha_{2}\right|=p_{2}}\left(\left.\int|| \xi_{1}\right|^{-n_{1}+\epsilon_{1}}\left|\xi_{2}\right|^{-n_{2}+\epsilon_{2}}\right. \\
& \left.\left.* \partial_{\xi_{1}}^{\alpha_{1}} \partial_{\xi_{2}}^{\alpha_{2}}\left(\xi_{1} \xi_{2} m_{i, j}\left(\xi_{1}, \xi_{2}\right)\right)\right|^{2} d \xi_{1} d \xi_{2}\right)^{1 / 2}
\end{aligned}
$$


Next, we use fractional integration twice on the variables $\xi_{1}$ and $\xi_{2}$ respectively. Let $1 / q_{1}=1 / 2+\epsilon_{1} / n_{1}$ and $1 / q_{2}=1 / 2+\epsilon_{2} / n_{2}$. Then

$$
\begin{array}{r}
\left|T_{i, j} h\left(x_{1}, x_{2}\right)-T_{i, j} h\left(0, x_{2}\right)-T_{i, j} h\left(x_{1}, 0\right)-T_{i, j} h(0,0)\right| \\
\leq C \gamma^{-\sigma}\left(M_{s} h^{2}(0)\right)^{1 / 2} \sum_{\left|\alpha_{1}\right|=p_{1}} \sum_{\left|\alpha_{2}\right|=p_{2}}\left(\int \left(\left.\int|| \xi_{2}\right|^{-n_{2}+\epsilon_{2}}\right.\right. \\
\left.\left.\left.*^{2} \partial_{\xi_{1}}^{\alpha_{1}} \partial_{\xi_{2}}^{\alpha_{2}}\left(\xi_{1} \xi_{2} m_{i, j}\left(\xi_{1}, \xi_{2}\right)\right)\right|^{q_{1}} d \xi_{1}\right)^{2 / q_{1}} d \xi_{2}\right)^{1 / 2}
\end{array}
$$

where $*^{2}$ denotes the convolution operator on the second variable. By Minkowski's inequality, the last inequality is less than

$$
\begin{aligned}
& C \gamma^{-\sigma}\left(M_{s} h^{2}(0)\right)^{1 / 2} \sum_{\left|\alpha_{1}\right|=p_{1}} \sum_{\left|\alpha_{2}\right|=p_{2}}\left(\int \left(\left.\int|| \xi_{2}\right|^{-n_{2}+\epsilon_{2}}\right.\right. \\
& \left.\left.\left.*^{2} \partial_{\xi_{1}}^{\alpha_{1}} \partial_{\xi_{2}}^{\alpha_{2}}\left(\xi_{1} \xi_{2} m_{i, j}\left(\xi_{1}, \xi_{2}\right)\right)\right|^{2} d \xi_{2}\right)^{q_{1} / 2} d \xi_{1}\right)^{1 / q_{1}} \\
& \leq C \gamma^{-\sigma}\left(M_{s} h^{2}(0)\right)^{1 / 2} \sum_{\left|\alpha_{1}\right|=p_{1}} \sum_{\left|\alpha_{2}\right|=p_{2}}\left(\int \left(\int\left|\partial_{\xi_{1}}^{\alpha_{1}} \partial_{\xi_{2}}^{\alpha_{2}}\left(\xi_{1} \xi_{2} m_{i, j}\left(\xi_{1}, \xi_{2}\right)\right)\right|^{q_{2}}\right.\right. \\
& \left.\left.d \xi_{2}\right)^{q_{1} / q_{2}} d \xi_{1}\right)^{1 / q_{1}} \\
& \leq C \gamma^{-\sigma}\left(M_{s} h^{2}(0)\right)^{1 / 2} \sum_{\left|\alpha_{1}\right|=p_{1}} \sum_{\left|\alpha_{2}\right|=p_{2}} 2^{j n_{2}\left(1 / q_{2}-1 / 2\right)} \\
& \cdot\left(\int\left(\int\left|\partial_{\xi_{1}}^{\alpha_{1}} \partial_{\xi_{2}}^{\alpha_{2}}\left(\xi_{1} \xi_{2} m_{i, j}\left(\xi_{1}, \xi_{2}\right)\right)\right|^{2} d \xi_{2}\right)^{q_{1} / 2} d \xi_{1}\right)^{2 / q_{1}} \\
& \leq C \gamma^{-\sigma}\left(M_{s} h^{2}(0)\right)^{1 / 2} \sum_{\left|\alpha_{1}\right|=p_{1}} \sum_{\left|\alpha_{2}\right|=p_{2}} 2^{j n_{2}\left(1 / q_{2}-1 / 2\right)} 2^{i n_{1}\left(1 / q_{1}-1 / 2\right)} \\
& \cdot\left(\iint \partial_{\xi_{1}}^{\alpha_{1}} \partial_{\xi_{2}}^{\alpha_{2}}\left|\left(\xi_{1} \xi_{2} m_{i, j}\left(\xi_{1}, \xi_{2}\right)\right)\right|^{2} d \xi_{1} d \xi_{2}\right)^{1 / 2} \\
& \leq C \gamma^{-\sigma}\left(M_{s} h^{2}(0)\right)^{1 / 2} 2^{\left(-p_{1}+1+n_{1} / q_{1}\right)} 2^{\left(-p_{2}+1+n_{2} / q_{2}\right)} .
\end{aligned}
$$

Hence

$$
V I \leq \sum_{i<0} \sum_{j<0} \operatorname{osc}_{R} T_{i, j} h \leq C \gamma^{-\sigma}\left(M_{s} h^{2}(0)\right)^{1 / 2} .
$$

Combining the above estimates, we conclude that

$$
T^{\#} f\left(x_{1}, x_{2}\right)=\left(M_{s} f^{2}(x)\right)^{1 / 2} .
$$

(i) is proved.

For the proof of (ii), we use duality. Let $U=W^{-1 /(p-1)}$. Then the dual space of $L_{W}^{p}\left(R^{n_{1}} \times R^{n_{2}}\right)$ is $L_{U}^{p^{\prime}}\left(R^{n_{1}} \times R^{n_{2}}\right)$. There exists a function $g \in L_{U}^{p^{\prime}}\left(R^{n_{1}} \times R^{n_{2}}\right)$ such that

$$
\left\|T_{m} f\right\|_{L_{W}^{p}}=\int T_{m} f \bar{g}=\int f \overline{T_{m} g} \leq\|f\|_{L_{W}^{p}}\left\|T_{m} g\right\|_{L_{U}^{p^{\prime}}} .
$$


It is easy to see that

$$
W^{2 /(2-p)} \in A_{p /(2-p)} \Longleftrightarrow U \in A_{p^{\prime} / 2}
$$

Applying (i),

$$
\left\|T_{m} g\right\|_{L_{U}^{p^{\prime}}} \leq C\|g\|_{L_{U}^{p^{\prime}}}
$$

where $U \in A_{p^{\prime} / 2}$. The Theorem is proved.

\section{REFERENCES}

1. L. Carleson, A counterexample for measures bounded for $H^{p}$ for the B-disc, Mittag Leffler report, No. 7, 1974.

2. R. Fefferman, Harmonic Analysis on Product Spaces, Annals of Math. 126 (1987), 109-130. MR 90e: 42030

3. J. Garcia-Cuerva and J. L. Rubio de Francia, Weighted norm inequality and Related Topics, North-Holland, 1985. MR 87d:42023

Department of Mathematics, Oregon State University, Corvallis, Oregon 97331

E-mail address: chen@math.orst.edu

Department of Mathematical Sciences, University of Wisconsin - Milwaukee, MilWAUKEE, WisCONSIN 53201

E-mail address: fan@csd.uwm.edu 